\title{
GESTÃO DO CONHECIMENTO ORGANIZACIONAL VISANDO À TRANSFERÊNCIA DE TECNOLOGIA: OS DESAFIOS ENFRENTADOS PELO NIT DA UNIVERSIDADE ESTADUAL DE SANTA CRUZ
}

\section{KNOWLEDGE MANAGEMENT AIMING TO TECHNOLOGY TRANSFER: THE CHALLENGES FACE BY THE TIC OF THE STATE UNIVERSITY OF SANTA CRUZ}

\author{
Luan Carlos Santos Silva*E-mail: luancarlosmkt@gmail.com \\ João Luiz Kovaleski* E-mail: kovaleski@utfpr.edu.br \\ Silva Gaia* E-mail: gaia@utfpr.edu.br \\ *Universidade Tecnológica Federal do Paraná, UTFPR, Ponta Grossa, PR.
}

\begin{abstract}
Resumo: O presente artigo traz para discussão reflexões acerca do entendimento de conhecimento organizacional, os modelos de teorias da criação e gestão de conhecimento que, em muitas abordagens, não levam em consideração a especificidade de cada organização, relacionamento intrínseco nos processos da dinâmica da inovação e sua contribuição para a transferência de tecnologia nos Núcleos de Inovação Tecnológica junto ao setor produtivo. A metodologia de pesquisa foi qualitativa e assumiu natureza descritiva. O método utilizado na pesquisa foi estudo de caso em um NIT de uma universidade pública no sul da Bahia. Finaliza, constatando que o NIT estudado está bem estruturado no que diz respeito à constituição dos NITs sob a ótica da Lei de Inovação Federal $n^{\circ} 10.973$ e da Lei de Inovação do Estado da Bahia $n^{\circ} 11.174$, existindo, porém, algumas barreiras para a criação e gestão do conhecimento que garantam a efetiva transferência de tecnologia.
\end{abstract}

Palavras-chaves: Criação do conhecimento. Transferência de tecnologia. Lei de Inovação. Núcleo de inovação tecnológica.

Abstract: The present article discusses the concept of organizational knowledge, and theory models for the creation and management of organizational knowledge that, in many approaches, do not take into account the specificity of each organization and the relationship. The paper analyses the innovation dynamics and the technology transfer from Technological Innovation Centers (TIC) to the productive sector. The methodology employed was qualitative and of descriptive nature. The method used in the research was a case study in a TIC well-structured in respect to the constitution of the TICs from the perspective of the Federal Innovation Law $n^{\circ} 10.973$ of the Innovation Law of the Bahia State $n^{\circ}$ 11.174. Barriers for knowledge creation and management that guarantee the effective technology transfer were identified.

Key-words: Creation Knowledge. Technology Transfer. Innovation Law. Technological Innovation Centers.

\section{INTRODUÇÃO}

Devido à alta competitividade do cenário atual gerada pela abertura de novos mercados e a exigência cada vez maior por produtos inovadores, as empresas 
tendem a buscar continuamente parcerias estratégicas visando à transferência de tecnologia com universidades ou centros de pesquisa de ponta, inovando sempre seus processos, produtos ou serviços. A interação com a era digital, oriunda da disseminação de novas tecnologias de informação e meios de comunicação, estreita significativamente, os obstáculos existentes nos mercados (EVANS e WURSTER, 1997).

Por um lado, as universidades brasileiras, de uma forma geral, têm estado há muito tempo afastadas das empresas nacionais, transferindo o desenvolvimento tecnológico ao mercado, como um objetivo menor ou até depreciativo, tendo seu crescimento na segunda metade do século $X X$ orientado para a formação de pessoal e para a produção de artigos científicos, no que foi, também de maneira geral, bem sucedida. Também o empresariado brasileiro tem-se afastado da universidade e da academia como um todo e, conquanto criativa para sobreviver a um mercado instável, aos ciclos de inflação, a insegurança jurídica e outros, tem relegado a último plano a introdução e o desenvolvimento de novas tecnologias em seus produtos. Este descompasso entre os setores produtores de conhecimento e potenciais transformadores deste conhecimento em produtos inovadores tem sido identificado cada vez mais como uma vulnerabilidade importante do país nesta era de crescente globalização e acelerado desenvolvimento de novos conhecimentos e mercados.

Para tal, o presente artigo foi desenvolvido no Grupo de Pesquisa em Gestão da Transferência de Tecnologia, no Programa de Pós-Graduação em Engenharia de Produção da Universidade Tecnológica Federal do Paraná (PPGEP/UTFPR). Este artigo traz para a discussão reflexões acerca do entendimento da criação de conhecimento organizacional e transferência de tecnologia nos Núcleos de Inovação Tecnológica (NIT), levando em consideração a especificidade de cada organização, região onde atuam e o relacionamento intrínseco nos processos de dinâmica da inovação e contribuição do conhecimento organizacional, para transferência de tecnologia, objetivando o desenvolvimento e socialização da inovação no setor produtivo. 
A metodologia utilizada para realização da pesquisa foi um estudo de caso em um NIT de uma universidade pública do Estado da Bahia. O objetivo principal deste artigo é mostrar a contribuição da gestão do conhecimento organizacional para a transferência de tecnologia no NIT, além de mostrar os desafios enfrentados pelo NIT na difusão da inovação na região.

O Núcleo de Inovação Tecnológica (NIT) tem-se mostrado adequado para viabilizar a transferência de tecnologia desenvolvendo a região onde estão inseridas. O NIT da Universidade Estadual de Santa Cruz (UESC) em particular, é uma instância ligada à Reitoria da Instituição de Ensino Superior (IES) e, tem por objetivo promover a inovação e a adequada proteção das invenções geradas nos âmbitos interno e externo da UESC e a transferência de tecnologia ao setor produtivo, visando integrá-la à comunidade e contribuir para o desenvolvimento cultural, tecnológico e social da região.

Recentemente, estabeleceu-se um movimento das universidades na direção do reconhecimento da necessidade de se superar este distanciamento das empresas. Houve, assim, o estabelecimento das Leis de Inovação federais e estaduais, que criaram incentivos para este relacionamento e demandaram o estabelecimento dos Núcleos de Inovação Tecnológica, para promover esta aproximação, além da proteção da propriedade intelectual na academia e nos setores e espaços sob sua influência. A UESC conta atualmente com um NIT institucionalizado, com Regimento aprovado na Resolução 05/2009 do CONSEPE e vinculado diretamente à Reitoria. A consolidação do NIT como instância importante para a inovação tecnológica na região e elemento de ligação entre a academia e o meio produtivo é oportuna e necessária para o desenvolvimento do sul da Bahia.

\section{REFERENCIAL TEÓRICO}

\subsection{Gestão do conhecimento organizacional}

No cenário atual, o processo de modernização no sistema empresarial, atrelado à qualidade, produtividade e novas tecnologias dos produtos e processos, 
está exigindo que empresas busquem sempre inovações em suas atividades no mercado alvo. Em função desses novos parâmetros na esfera empresarial, muitas organizações de vários setores têm se preocupado em repensar suas antigas formas de produção.

Percebe-se que estas mudanças criam a necessidade para geração de inovações contínuas a serem estimuladas e geradas pelos indivíduos nas organizações, com o objetivo de se manterem operantes no atual ambiente competitivo. Tais inovações, necessárias e fundamentais, podem surgir a partir de brainstormings ou ideias inesperadas e imprevisíveis, no entanto, oriundas tacitamente na mente das pessoas que compõem as diversas organizações.

Todavia, para converter o conhecimento tácito em conhecimento explícito e, a partir daí, poder criar inovações, os indivíduos devem ser motivados a participar continuamente dos processos importantes de suas atividades executadas, e principalmente, a compartilharem seus conhecimentos tácitos, na forma de experiências vividas na ambiente organizacional. A gestão do conhecimento organizacional é uma ferramenta imprescindível para a geração de inovações nas organizações em todas as etapas de seus processos, desde o compartilhamento do conhecimento tácito entre os indivíduos, até a globalização deste conhecimento por toda a organização (NONAKA e TAKEUCHI, 1995, p.68).

Para Szezerbicki et. al (2006), a gestão do conhecimento aparece para reorganizar e potencializar os fatores de produtividade, inovação, competitividade e relacionamento das empresas no âmbito em que operam.

Desta forma, esta gestão atenta-se em fornecer capacitação às pessoas dentro das empresas, estimulando-as a tomarem decisões diariamente o que, em conjunto, determinarão o sucesso ou o fracasso de um negócio (REIS, MILAN, 2009).

Segundo Sveiby (1998), a conceituação de gestão do conhecimento apareceu em meados dos anos 90, tendo como objetivo primordial uma criação de valor no âmbito interno das empresas, almejando a otimização dos recursos disponíveis, seja tecnológico, humano ou financeiro, sendo este exequível por meio da aprendizagem entre os indivíduos na empresa. 
Lacombe (2005) salienta que a gestão do conhecimento no âmbito organizacional nas últimas décadas atingiu um ponto importantíssimo nas empresas, onde ser competitivo em suas atividades no mercado atuante é indispensável para uma excelente criação e gestão deste conhecimento oriundo de cada indivíduo na organização em suas diversas formas de abordagem. Somente desta forma ela pode tornar-se competitiva e sustentável no mercado.

A inovação e a gestão do conhecimento nos processos de uma empresa podem ser compreendidas como um aglomerado de empenhos gerados pelos indivíduos almejando criar, adquirir, transformar, aplicar e posteriormente proteger a criação do conhecimento gerada pela organização visando manter-se competitiva no mercado atuante (DAVENPORT e PRUSAK, 2003; FLEURY e OLIVEIRA, 2001; GOLD, MALHOTRA e SEGARS 2001).

E para que a inovação seja possível na ambiência da organização, o conhecimento deve ser estimulado e desenvolvido em todas as etapas do processo de gestão. O conhecimento pode ser compreendido como uma ligação de práticas vivenciadas pelo homem, como: valores, informação contextual e ideias geradas. Tais experiências possibilitam ao indivíduo uma estrutura para a avaliação e implementação de novas práticas e informações, tendo sua origem e aplicação na mente do homem. Nas empresas, o conhecimento não se restringe apenas em documentos ou guias de normas e manuais de gestão, mas também está nas rotinas, processos e formas de conduzir o trabalho dos indivíduos (DAVENPORT e PRUSAK, 2001; SANTIAGO Jr 2004).

Sobre a compreensão de criação do conhecimento e dinâmica dessa criação, Nonaka e Takeuchi (1995) propõem um Modelo de Conversão de Conhecimento. Os mesmo salientam que a criação do conhecimento organizacional é um processo em espiral, que começa no nível individual e vai subindo, ampliando comunidades de interação que cruzam fronteiras entre seções, departamentos, divisões e organizações.

A criação do conhecimento organizacional, pois, deve ser entendida como um processo que amplia "organizacionalmente" o conhecimento criado pelos indivíduos, cristalizando-o como parte da rede de conhecimento da organização. Esse processo ocorre dentro de uma "comunidade de interação" em expansão, que atravessa níveis e fronteiras interorganizacionais. (NONAKA e TAKEUCHI, 1995). 
A espiral do conhecimento SECI, pode ser visualizada na figura a seguir:

Figura 1 - Espiral SECl através das Conversões de Conhecimento em diferentes níveis

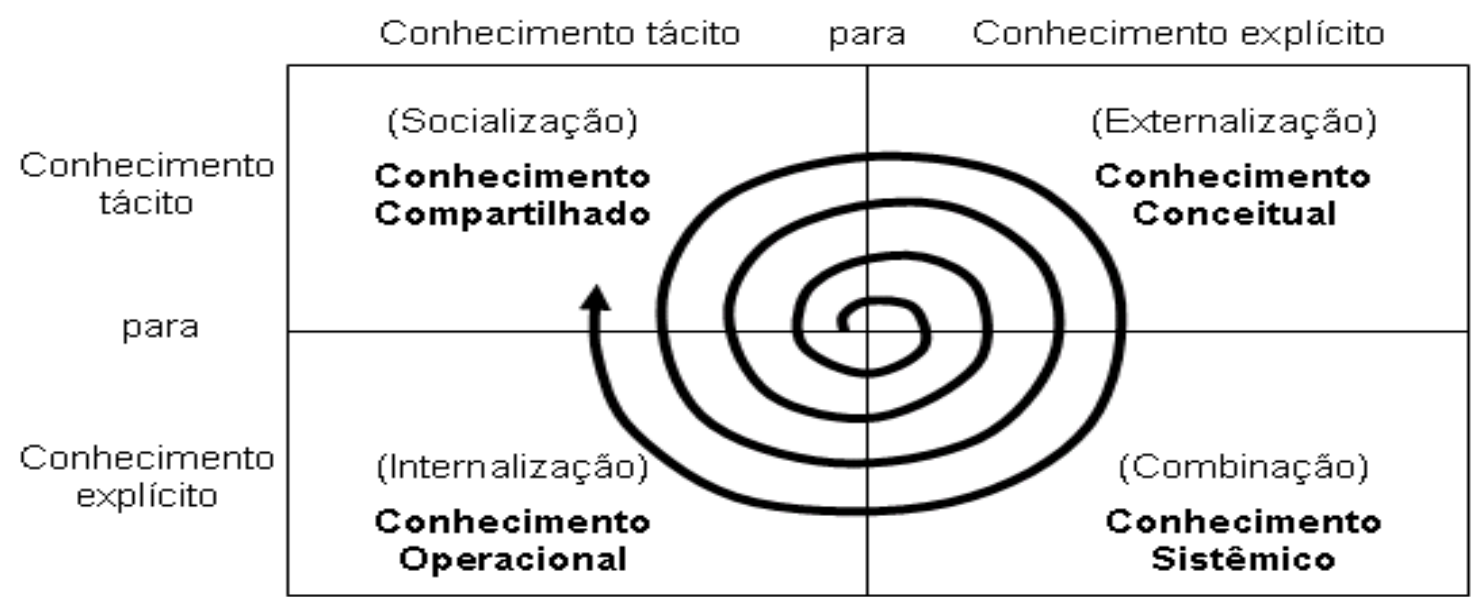

Fonte: Nonaka e Takeuchi (1995)

Conforme Nonaka e Takeuchi (1995), no processo de socialização, os indivíduos conversam e repassam informações entre si, transferindo o conhecimento tácito de um, para o conhecimento tácito do outro. Esse processo se dá através do compartilhamento de experiência entre pessoas, e possibilita a geração de conhecimento tácito a partir do tácito. A experiência é o principal elemento que deve estar presente nesse processo de conversão, pois sem alguma forma de experiência compartilhada, é muito complicado para uma pessoa poder entender o raciocínio da outra. Nesta etapa de socialização desenvolve-se o chamado conhecimento compartilhado.

Ainda no mesmo sentido Nonaka e Takeuchi (1995) salientam que na externalização, o conhecimento tácito recebido é transformado em conhecimento explícito, através de conceitos, que podem ser inclusive documentados. Seja através da comunicação falada ou escrita, o conhecimento tácito pode ser convertido em conhecimento explícito através de metáforas, analogias, conceitos, hipóteses ou modelos. O processo de externalização gera um tipo de conhecimento chamado conhecimento conceitual.

No terceiro ponto da espiral do conhecimento, Nonaka e Takeuchi (1995) relatam sobre a combinação, onde os conhecimentos explícitos externalizados são comparados com outros conhecimentos explícitos já existentes sobre um 
determinado assunto, analisando-se aí as convergências e divergências evidenciadas.

O processo de combinação gera um tipo de conhecimento chamado conhecimento sistêmico, ocorrido através de agrupamento do conhecimento (classificação e sumarização), ou processamento de diferentes conhecimentos explícitos.

E por último, Nonaka e Takeuchi (1995) salientam que o novo conhecimento explícito gerado pela combinação volta a ser tácito, através do processo de internalização, o qual promove a certeza de que o indivíduo que recebeu os novos conhecimentos, verdadeiramente aprendeu. Este processo pode ser caracterizado pelo aprendizado através da prática.

O processo de internalização gera um tipo de conhecimento chamado conhecimento operacional, ocorrendo por meio de leitura ou visualização e estudo individual de documentos de diferentes formatos e modelos, tais como textos, imagens etc., prática individual, reinterpretar e reexperimentar, individualmente, vivências e práticas, dentre outros pontos.

Contudo, o esquema da espiral do conhecimento completa este ciclo de conversão do conhecimento, estimulando a certeza de que realmente ocorreu a efetiva criação do conhecimento, e de que houve efetivamente um aproveitamento adequado e satisfatório do conhecimento criado, podendo este vir a promover inovações a partir de sua implementação. Contudo, apenas com este ciclo completo, é que se desenvolve a gestão do conhecimento, possibilitando neste processo a dinâmica da inovação organizacional.

\subsection{Transferência de tecnologia}

A transferência de tecnologia passou a ser um meio eficaz para a disseminação da inovação e do conhecimento, sendo uma alternativa competitiva para as empresas na busca não somente da exploração dos recursos internos para utilização de novas tecnologias, mas também, na aquisição de parceiros externos, 
no incremento de novas tecnologias, consistindo nisso, a tarefa básica da transferência de tecnologia.

Conforme Cysne (2005) a transferência de tecnologia teve seu marco durante a revolução industrial, pois foram desenvolvidas tecnologias na Inglaterra que, logo em seguida, foram transferidas paras as indústrias da América, Europa, e Rússia. Esse processo de transferência de tecnologia permeou por todo o século 19, sendo que no século 20 seu desenvolvimento fora significativo e também continuou sua expansão de atividades no início deste século.

A definição de transferência de tecnologia pode ser interpretada como um processo de aquisição, desenvolvimento e uso de conhecimentos tecnológicos pelos indivíduos que o geraram (LIMA, 2004). No entanto, entende-se como um processo de implementação de novas tecnologias desenvolvidas para uma ambiência que não possui a mesma tecnologia, com a finalidade de melhorar a capacidade da organização de executar as atividades (HSU, 2008; MINBAEVA et al., 2003; CUMMINGS e TENG, 2003, DAVENPORT e PRUSAK, 2003; FITZEK, 2002; FRANK e ECHEVESTE, 2011).

Deitos (2002) apresenta as etapas do processo de transferência de tecnologia de forma bastante simples e de fácil entendimento para os envolvidos no processo, compreendendo 6 etapas:

1) Seleção da tecnologia a ser utilizada pela empresa;

2) Seleção dos fornecedores da tecnologia;

3) Negociação para a aquisição da tecnologia;

4) Realização do processo de transferência da tecnologia;

5) Assimilação da tecnologia transferida;

6) Implementação e adaptação e melhoramento da tecnologia.

Durante o processo de transferência de tecnologia várias informações técnicas são envolvidas, desde o processo de desenvolvimento dos produtos e processos, mercados, fornecedores, uso das tecnologias, bem como a parte metodológica para cada etapa de implementação da nova tecnologia pela organização. A metodologia por sua vez, pode ser compreendida pelo meio no qual a tecnologia será transferida para o usuário final, desenvolvendo-o para que a sua 
implementação seja efetivada garantindo a completa transmissão dos conhecimentos entre os envolvidos (ESCORSIM, 2006).

As pessoas envolvidas no processo da transferência de novas tecnologias, devem implementar uma metodologia de forma natural e direta, observando pontos de controle bem como seu devido monitoramento (ROMANENKO; SANTOS e AFONSO, 2007).

Além disso, o correto seria que essas mesmas pessoas envolvidas na implementação das novas tecnologias alinhassem com as necessidades das estratégias da organização, observando as capacidades operacionais e estruturais, minimizando seus custos e garantindo a correta execução da operação (BROWN; SQUIRE e BLACKMON, 2007).

Na visão de Bozarth (2006), ao fazer uma transferência e implementação de tecnologias na organização, um minucioso monitoramento de todas as etapas faz-se necessário para que tudo transcorra conforme o planejado. Para tanto, as atividades de suporte garantem que a transferência de tecnologia ocorra com sucesso e seja realmente efetivada. Sobre esta característica, algumas pesquisas destacam que quanto maior for a complexidade das atividades, maior é a necessidade de aproveitar os conhecimentos das equipes, pois precisa-se aproveitar mais os componentes e ideias de outras atividades, fazendo com que a transferência assuma maior relevância (CHAPMAN e HYLAND, 2004; AKGÜN et al., 2005; EDMONDSON e NEMBHARD, 2009; KLEINSMANN et al., 2010; PICININ, KOVALESKI e REIS, 2010).

Para Niedergassel e Leker (2010) a cooperação estabelecida entre as universidades e o setor produtivo pode, assim, auxiliar na busca de novas invenções que trazem estímulos importantes para o desenvolvimento de inovações e transferência de tecnologias.

Contudo, muitos outros autores destacam que o sucesso das empresas depende cada vez mais da sinergia obtida dos diferentes projetos de produtos através do aprendizado e a transferência de conhecimentos resultantes da interação e integração desses projetos (BOURGEON, 2007; MARSH e STOCK, 2006; ANTONI et al., 2005; CORSO e PAVESI, 2000). 


\subsection{Lei de inovação e transferência de tecnologia}

Conforme Matias-Pereira e Krugliaskas (2005), a lei de inovação constitui-se numa etapa importante para que se promova maior envolvimento entre as esferas que desenvolvem e utilizam o conhecimento, sendo uma ambiência compreendida por centros de pesquisa, universidades, também por empresas e empreendimentos, cooperativas entre outros. A lei $n^{\circ} 10.973$, de 02/dez/04, ou Lei da Inovação, em termos gerais compreende os incentivos à inovação e à pesquisa científica e tecnológica no ambiente produtivo, representando um marco importante no cenário competitivo brasileiro. Importante ressaltar, que na Lei de Inovação, em seu artigo $1^{\circ}$, nos termos dos artigos 218 e 219 , dispõe de medidas de incentivo à inovação e à pesquisa científica e tecnológica no ambiente produtivo, como:

Constituição Federal: "Art. 218 - O Estado promoverá e incentivará o desenvolvimento científico, a pesquisa e a capacitação tecnológicas (e seus parágrafos $1^{\circ}, 2^{\circ}, 3^{\circ}, 4^{\circ}$ e $5^{\circ}$ )"; e "Art. 219 - O mercado interno integra o patrimônio nacional e será incentivado de modo a viabilizar o desenvolvimento cultural e socioeconômico, o bem-estar da população e a autonomia tecnológica do País, nos termos de lei federal". A lei faz referência e de fato implementa o disposto nos dois artigos constitucionais acima citados no que tange ao papel do Estado de incentivar o desenvolvimento científico, a pesquisa e a capacitação tecnológicas e de reconhecer no mercado nacional o destinatário do incentivo previsto, de forma que a partir dele, o país possa buscar a sua autonomia tecnológica e promover o bem-estar da população. (MATIAS-PEREIRA e KRUGLIASKAS, 2005).

Conforme Barbosa (2006, p.34), de modo abrangente, a lei procura atender particularmente aos objetivos de incentivar a pesquisa científica e tecnológica e a inovação; incentivar a cooperação entre os agentes de inovação; facilitar a transferência de tecnologia; aperfeiçoar a gestão das instituições acadêmicas; servir de estímulo aos pesquisadores; estimular a mobilidade dos pesquisadores; estimular a formação de empresas de base tecnológica; e, estimular o investimento em empresas inovadoras.

Outro ponto importante da Lei de Inovação foi no que tange à proteção do conhecimento. A lei determina que cada Instituto de Ciência de Tecnologia (ICT), constitua um Núcleo de Inovação Tecnológica (NIT) próprio ou em associação com outras ICTs. Isso possibilitou que muitas universidades e centros de pesquisas 
criassem seus NITs e difundissem a inovação em suas regiões, visando à transferência de tecnologia junto ao setor produtivo.

Para Martins (2010), o NIT desempenha papel fundamental na relação entre as empresas e as instituições de pesquisa e desenvolvimento, uma vez que busca atender as demandas dos atores envolvidos, adotando as estratégias e diretrizes previstas nas Políticas Públicas de Inovação e Tecnologia, contribuindo para o desenvolvimento da inovação no Brasil, bem como estimulando a transferência de tecnologia entre os ICTs e o setor produtivo.

Nessa vertente, a lei faculta aos ICTs celebrar contratos de transferência de tecnologia e de licenciamento de patentes de sua propriedade, prestar serviços de consultoria especializada em atividades desenvolvidas no âmbito do setor produtivo, assim como estimular a participação de seus funcionários em projetos onde a inovação seja o principal foco.

A lei de Propriedade Intelectual do Brasil ( $n^{\circ}$ 9.279/96) faculta ao Instituto Nacional de Propriedade Intelectual (INPI) celebrar a averbação de 6 tipos de contratos de transferência de tecnologia, dentre eles: Exploração de patentes, desenho industrial, fornecimento de tecnologia, prestação de serviço de assistência técnica e científica, franquias, e uso de marcas.

Conforme dados do fechamento anual de transferência de tecnologia do INPI, somente no ano de 2009 foram depositados e certificados 1.739 contratos de transferência de tecnologia no INPI aqui no Brasil. Isto representa um crescimento de $18 \%$ comparado ao ano de 2008; o Estado da Bahia em particular, teve 51 contratos depositados e certificados, o que representa um pequeno percentual de $4 \%$ no ano de 2009.

Os empresários e gestores atuais possuem um vasto sistema de incentivo e formas de implementarem suas ações, objetivando cada vez mais estimular e desenvolver a inovação em suas produções e fazer uso dos mecanismos de transferência de tecnologia garantindo sempre a proteção adequada do conhecimento gerado. 


\section{METODOLOGIA}

A pesquisa foi qualitativa e assumiu natureza descritiva, conforme descrito por Cervo \& Bervian (1996), Marconi \& Lakatos (1996) e Perin et al. (2002). O método utilizado na pesquisa foi estudo de caso (GIL, 2002; TRINDADE, 2003).

No primeiro momento, a pesquisa consistiu da análise de fontes secundárias, tais como artigos nacionais e internacionais, livros, resoluções, leis, etc.

No segundo momento, as referências analisadas na revisão de literatura serviram como base para a coleta de informações diretamente com o objeto de estudo, num período de 6 meses, de junho a dezembro de 2010.

Nesta etapa de 6 meses, houve um período de observação mais detalhado junto ao NIT-UESC, onde observamos a forma de planejamento e execução das atividades, articulações entre os membros internos e os demais departamentos da universidade, ações estratégicas junto ao setor produtivo. Foram analisadas as atividades das comissões de propriedade intelectual, informações tecnológicas, mas com ênfase maior na comissão de transferência de tecnologia, analisando principalmente a forma de criação de conhecimento.

Atualmente o NIT-UESC conta com 32 pessoas envolvidas em suas atividades, sendo: 2 gestores, o coordenador geral e vice-coordenador, 7 bolsistas de produtividade, com bolsa do CNPq e FAPESB, 3 representantes das comissões (Transferência de Tecnologia, Propriedade Intelectual e Informações Tecnológicas), e mais 20 representantes dos departamentos da universidade. Para cada departamento tem um representante principal e mais um suplente, conforme estabelecido em 2009 na Resolução 05 do CONSEPE.

Os 10 departamentos da UESC são: Departamento de Ciências Exatas e Tecnológicas (DCET), Departamento de Ciências Agrárias e Ambientais (DCAA), Departamento de Administração e Ciências Contábeis (DCAC), Departamento de Ciências Biológicas (DCB), Departamento de Ciências Econômicas (DCEC), Departamento de Ciências da Educação (DCIE), Departamento de Ciências da Saúde (DCS), Departamento de Ciências Jurídicas (DCIJUR), Departamento de Filosofia e Ciências Humanas (DFCH) e Departamento de Letras e Artes (DLA). 
Após este período de observação direta, foi elaborado um questionário qualitativo, contendo 5 questões-chaves que respondessem os objetivos da pesquisa junto ao NIT. Foram selecionadas para entrevistas pessoas com posições estratégicas, por existir algumas informações de acesso praticamente irrestrito. As entrevistas com os gestores e demais envolvidos no processo de transferência de tecnologia possibilitou maior autenticidade na capacidade da análise das informações coletadas referentes ao cenário real.

A seleção dos entrevistados aconteceu com uma amostra não probabilística intencional. Foram selecionadas todas as pessoas do NIT que atuam diretamente com as atividades de transferência de tecnologia, abrangendo assim a coordenação geral do NIT.

O questionário aplicado foi não estruturado (aberto), as entrevistas ocorreram durante 1 (uma) semana no Departamento de Pós-graduação da UESC, onde se encontra a sede do NIT. As perguntas foram aplicadas por meio do questionário, os entrevistados anotaram suas observações de acordo com as perguntas.

Abaixo se encontra $o$ roteiro das questões elaboradas e aplicadas nas entrevistas.

1. Quais infraestruturas disponíveis existem para atender os sistemas locais de inovação e capacidade instalada de inovação, ciência de tecnologia (ICT) visando à transferência de tecnologia da UESC?

2. Quais são os planos de expansão visando à transferência de tecnologia entre universidade e setor produtivo da região?

3. Quais parcerias e articulação interinstitucional visando à transferência de tecnologia?

4. Como são planejadas e executadas as formas de criação de conhecimento visando à transferência de tecnologia?

5. Quais são as ações e quantos contratos de transferência de tecnologia foram efetivados junto ao Instituto Nacional de Propriedade Industrial?

Após a aplicação do questionário, os dados foram analisados separadamente conforme as respostas dos entrevistados, depois foram retiradas as principais ideias de cada resposta e agrupadas em uma única resposta. 


\subsection{Descrição do NIT pesquisado}

O NIT-UESC foi estruturado visando atender as demandas locais da universidade e do setor produtivo, atendendo às Leis de Inovação, tanto federal (Lei $n^{\circ}$ 10.973), como estadual (Lei $\left.n^{\circ} 11.174\right)$.

A UESC teve seu Núcleo de Inovação Tecnológica institucionalizado desde maio de 2009 (Resolução 05 do CONSEPE), estando ligado à Reitoria e tendo seu conselho formado por um representante de cada Departamento, mais um representante da PROPP (Pró-Reitoria de Pós Graduação e Pesquisa). Estes 11 representantes se dividem nas funções de coordenador e vice e nas comissões de Propriedade Intelectual (P.I.), Transferência de Tecnologia (T.T), e Informações Tecnológicas (I.T.). A UESC conta também com quatro Empresas Júnior (nas áreas de Computação, Engenharia de Produção, Administração e Línguas Estrangeiras Aplicadas a Negociações Internacionais), a incubadora da agroindústria e a incubadora de empreendimentos solidários, além de ter ex-alunos atuando na incubadora do CEPEDI, o INETI.

Está localizado na Universidade Estadual de Santa Cruz (UESC), situado no sudeste da Bahia, no município de llhéus e tem sua abrangência de atuação na Região Sul. É a única instituição pública de ensino superior desta região, composta pelas regiões econômicas do Litoral Sul, com 53 municípios, e do Extremo Sul, com 21 municípios, totalizando 74 municípios. Essa área com $53.931 \mathrm{Km} 2$ equivale a 9,61\% da área do Estado e engloba uma população estimada de 2.450 .000 habitantes, correspondendo a $19,5 \%$ da população baiana. Do ponto de vista econômico o Produto Interno Bruto - PIB em despesas correntes, no ano de 2009 das regiões Litoral Sul e Extremo Sul contribuíram com 15,2\% do total arrecadado no Estado, conforme informações disponíveis na Fundação de Amparo à Pesquisa da Bahia (FAPESB).

Está localizada também em uma das mais extensas áreas de Mata Atlântica preservada do Nordeste brasileiro. Tem um entorno característico: região agrícola terra do cacau - com uma área de 600 mil hectares de cacauais num sistema de produção secular que permitiu a conservação dos estratos superiores das formações 
florestais, e manteve um patrimônio genético de valor inigualável, conferindo-lhes a característica de alta diversidade florística e faunística.

A região, que também conta com empresas do setor alimentício e têxtil deve abrigar, nos próximos anos, novo porto exportador off-shore, aeroporto internacional, num complexo multimodal completado pela ferrovia Oeste-Leste, que trará os produtos desde Tocantins até Ilhéus, passando por áreas de produção agrícola e mineradoras do interior da Bahia. A GASENE (Gasoduto que levará gás do Nordeste ao Sudeste) teve sua primeira central de distribuição recentemente instalada em Itabuna, a $15 \mathrm{~km}$ da UESC.

\section{RESULTADOS E ANÁLISE DOS DADOS}

A seguir, uma narrativa das principais análises das perguntas feitas junto aos entrevistados, entre elas: infraestrutura disponível para atender os sistemas locais de inovação e capacidade instalada de inovação, ciência de tecnologia (ICT) visando à transferência de tecnologia; plano de expansão visando à transferência de tecnologia entre universidade e setor produtivo; parcerias e articulação interinstitucional visando à transferência de tecnologia; criação de conhecimento visando à transferência de tecnologia; e, contratos de transferência de tecnologia.

\subsection{Infraestrutura disponível para atender os sistemas locais de inovação e capacidade instalada de inovação, ciência de tecnologia (ICT) visando à transferência de tecnologia}

No entendimento dos entrevistados, os programas de pesquisas existentes são capazes de estimular o desenvolvimento de novas tecnologias que garantam novas inovações, e futuras transferências de tecnologias junto ao setor produtivo.

A UESC conta hoje com um total de 98 grupos de pesquisa certificados e cadastrados pela Instituição no CNPq, divididos nas diversas áreas do conhecimento, destacando-se as biológicas e agrárias. No âmbito da pesquisa e da pós-graduação, o Planejamento Institucional da UESC adota como estratégias a 
melhoria qualitativa dos cursos de pós-graduação garantindo a indissociabilidade entre ensino, pesquisa e extensão; a interação do ensino com a área de abrangência da UESC, a promoção da integração entre graduação e pós-graduação, a implantação e consolidação de grupos interdisciplinares de pesquisa; a consolidação dos cursos de pós-graduação implantados e na dinamização da infraestrutura de produção do conhecimento (laboratórios, bibliotecas e Centro de Processamento de Dados).

O Núcleo de Inovação Tecnológica da UESC dispõe de sala com 5 computadores, data-show, câmera fotográfica digital, livros diversos, 3 notebooks e impressora multifuncional. Dispõe também de equipamento para videoconferência, incluindo codec, câmera e tela de 42" e dispositivo de audioconferência.

\subsection{Plano de expansão visando à transferência de tecnologia entre universidade e setor produtivo}

$\mathrm{Na}$ visão dos entrevistados, o principal plano de expansão do NIT para a UESC visando à transferência de tecnologia está ligado à criação do Parque Tecnológico. Este parque tecnológico uma vez consolidado, deve ter atuação em duas vertentes principais: Tecnologias da Informação e Comunicação e Biotecnologia. Como há um polo industrial consolidado no setor de TICs, julgamos que esta vertente deve ser implantada inicialmente, sendo que a implantação da vertente biotecnológica está em estudos e será apresentada num documento complementar.

Para Steiner et al. (2008, p. 9) os Parques Tecnológicos aliando-se a essas novas formas de apoio à inovação são mecanismos já consolidados mundialmente como plataforma de desenvolvimento de ciência, tecnologia e inovação (C,T\&I) e de empresas inovadoras. Envolvem a aglutinação geográfica e mecanismos funcionais de cooperação entre os setores acadêmico e produtivo, potencializando a transferência e o investimento em conhecimento.

Os principais envolvidos na criação deste Parque Tecnológico seriam as empresas do Polo, representadas por seu sindicato (SINEC), o poder público e as 
principais Instituições de Ciência e Tecnologia (ICT) da região com histórico de parceria com estas mesmas empresas, o CEPEDI e a UESC.

Estas duas entidades têm agido em conjunto, como instituições acadêmicas próximas e parceiras das empresas do Polo e esta proximidade caracteriza a iniciativa de criação de um Parque Tecnológico local como oportuna e urgente.

O Sindicato das Indústrias de Aparelhos Elétricos, Eletrônicos, Computadores, Informática e Similares de llhéus e Itabuna (SINEC) têm representado as empresas da região desde novembro de 1998. Ele surgiu com o objetivo de representar e lutar pelos interesses dos empresários que acreditam no potencial econômico do setor. Ao longo desses anos, o SINEC vem buscando desenvolver novas propostas para o Polo de Informática de Ilhéus, como a melhoria da estrutura física e a ampliação da oferta de mão de obra qualificada, que contribui para que as empresas consigam absorver o trabalho dos profissionais da cidade. Para a constituição do Parque é imprescindível a criação de uma instância de gestão compartilhada entre administração pública local (prefeitura e governo do estado), academia e empresas, com pessoal especializado.

A cooperação estabelecida entre universidade e o setor produtivo pode, assim, auxiliar na busca de novas invenções que tragam estímulos importantes para o desenvolvimento de inovações (NIEDERGASSEL e LEKER, 2010).

A cooperação entre universidade e empresas acontece geralmente por conta de objetivos específicos, frequentemente de curto a médio prazo. São, contudo, possíveis devido a ações de capacitação física e de pessoas estratégicas de parte a parte.

Os termos e temas das ações de cooperação específicas entre empresas individuais e pesquisadores ou grupos podem ser, inclusive, objeto de sigilo. Da mesma maneira, planos de ação em pesquisa e crescimento de grupos e departamentos acadêmicos podem não envolver necessariamente demandas sociais e de empresas. Por outro lado, a universidade e o setor produtivo podem e devem encontrar vastas áreas de cooperação e decisões dos dois segmentos ou podem apenas ser positivamente afetadas se houver continuado conhecimento das necessidades e capacidades dos dois lados. As particularidades de cada entidade 
específica não impedem ações e demandas coletivas, estruturantes e a convergência de ações.

Neste sentido, é importante que haja uma instância para troca de informações a respeito das oportunidades estratégicas para que também o Estado possa ser mobilizado para agir como facilitador destas parcerias.

\subsection{Parcerias e articulação interinstitucional visando à transferência de tecnologia}

Conforme os entrevistados, a UESC possui parcerias em projetos de inovação com empresas localizadas na região e algumas de fora do Estado, em especial as empresas do Polo de Informática de Ilhéus (Bitway, Nortcom, Eclipse, Ibracomp, Accept), do setor alimentício (Mais Cacau), borracha (Michelin), entre várias outras ativas ou em elaboração. Há também contatos e convênios em elaboração com prefeituras locais que preveem ações de extensão e inovação, além de várias parcerias com instituições acadêmicas em pesquisa nos âmbitos estadual, nacional e internacional. Com base nas parcerias regionais, em especial com o CEPEDI e empresas do Polo e sua representação (o SINEC Sindicato que representa estas empresas), foram iniciados contatos envolvendo a Secretaria de Indústria, Comércio e Mineração e Prefeitura de Ilhéus para o planejamento de um futuro Parque Tecnológico local. Este empreendimento não tem ainda um cronograma definido e sua definição fará parte das atividades da equipe deste projeto.

O NIT-UESC tem agido em parceria com os NITs das outras universidades estaduais da Bahia e com a UFBA, especialmente no que se refere às ações de formação de pessoal coordenadas pela Diretoria de Inovação da FAPESB. Tem crescido também o contato com as outras instâncias promotoras da Inovação nas ICTs, em especial através da Rede NIT-Nordeste e do FORTEC. No âmbito local, há parcerias sendo elaboradas com as prefeituras de Ilhéus e Itabuna para uma série de questões. Há também as colaborações e cooperações técnicas com o Sindicato 
das Indústrias de Aparelhos Elétricos, Eletrônicos, Computadores, Informática e Similares de Ilhéus e Itabuna (SINEC).

O NIT-UESC tem buscado alinhar-se junto ao setor produtivo e órgãos importantes ligados à atividade econômica da região sul da Bahia, objetivando estabelecer a cooperação em universidade-empresa. Apoiando-se nessa linha de pensamento, Debackere e Veugelers (2005), salientam que o desenvolvimento de uma estrutura apropriada para a cooperação universidade-empresa demanda uma atenção especial sobre os interesses da universidade e, sobretudo, empresariais.

\subsection{Criação de conhecimento visando à transferência de tecnologia}

Para os entrevistados, o NIT-UESC no que tange à socialização, externalização, combinação e internalização do conhecimento organizacional (NONAKA e TAKEUCHI, 1995), ainda é escasso o uso sistemático das etapas citadas, porém buscam sempre transmitir aos membros as informações necessárias sobre as atividades internas e externas do NIT.

Uma das grandes barreiras para a criação efetiva do conhecimento organizacional, citada pelos entrevistados, é que não possuem um quadro efetivo de funcionários no NIT, apenas têm bolsistas, conforme prevê a lei de inovação, e tem contado basicamente com a contratação de bolsistas através dos editais ofertados.

Os bolsistas são capacitados em cursos, palestras, workshops, oficinas ligadas à inovação, tanto na Bahia como em outros Estados, mas quando o projeto de pesquisa chega ao fim, todo o conhecimento adquirido é levado consigo, e quando outro vem ocupar seu lugar tem que passar por toda capacitação acima mencionada.

O NIT-UESC têm uma página institucional e um blog próprio, com boletim de oportunidades impresso quinzenalmente. Há a intenção de criar, em médio prazo, portal de inovação com material abundante e mecanismo para facilitar a interligação entre as demandas e ofertas de inovação, com mapeamento de competências internas, conexões e parcerias estabelecidas, bem como explicação sobre os mecanismos para facilitação ou financiamento destas parcerias. 
Conforme o entrevistado, as principais ações a serem promovidas pelo Estado da Bahia para maior fortalecimento dos NITs são a regulamentação da Lei de Inovação da Bahia e a implantação de cargos e funções para que os núcleos não fiquem na vinculação de bolsas das agências de fomento.

\subsection{Contratos de transferência de tecnologia}

Atualmente o NIT-UESC não depositou nenhum contrato de transferência de tecnologia no INPI, desde seu início em 2009 (Resolução 05 do CONSEPE), mas buscou mecanismos para que isso seja realmente efetivado, conforme visão dos entrevistados.

\section{CONCLUSÕES}

As empresas não estão alheias às mudanças que estão ocorrendo no atual cenário competitivo, porém, os gestores não têm dado o devido tratamento para as questões relacionadas às especificidades de cada organização, aos indivíduos para fomento da criação de conhecimento organizacional, processo da dinâmica da inovação e transferência de tecnologia.

Para enfrentar este novo desafio, elas precisam estar sempre inovando e adquirindo sucessivamente novos conhecimentos organizacionais através de parcerias estratégicas para poderem estar sempre apresentando uma postura competitiva, o que são tarefas complexas para países em processo de desenvolvimento, como o Brasil. Esses desafios com o tempo são difíceis de combater pela falta de consciência dos empresários pela inovação e proteção da propriedade intelectual gerada, e também pela ausência de incentivo de mestres e doutores nas indústrias. (BRITO-CRUZ, 1999; CASSIOLATO \& LASTRES, 2005; CHESNAIS, 1998; EDLER, KRAHMER, \& REGER, 2002; FLEURY, 1999; FREEMAN, 1991; MOTOHASHI, 2005; SEGATTO-MENDES, 2002; SUTZ, 2000).

Para tanto, é necessário que o NIT crie um ambiente propício à aprendizagem organizacional, flexível e sem estruturas hierárquicas rígidas. O mesmo pode ser 
uma excelente alternativa para as empresas na difusão da inovação que garanta a proteção do conhecimento gerado, bem como a efetivação da transferência de tecnologia junto ao INPI e o setor produtivo. A criação da Lei Estadual de Inovação possibilitou uma maior estruturação dos núcleos de inovação tecnológica.

O NIT-UESC desde 2009 possui uma excelente estrutura, tem buscado boas ações estratégicas, porém nenhum contrato de transferência de tecnologia foi depositado junto ao INPI. Observa-se, no que tange à criação e gestão de conhecimento proposto pelos especialistas, que ainda não tem um sistema eficaz de criação e gestão do conhecimento, inviabilizando ações tomadas junto ao núcleo que poderiam ser mais bem trabalhadas com membros e departamentos envolvidos dentro da universidade e o setor produtivo.

A falta de uma estrutura de criação de conhecimento impactou diretamente na difusão da inovação junto à universidade e setor produtivo regional, impossibilitando ações voltadas à transferência de tecnologia, seja por meio de licenciamento de patentes, prestação de serviços de assistência técnica e científica, ou fornecimento de tecnologia.

Apesar de o NIT pesquisado ter sido fundado em 2009, no Brasil a Lei de Inovação facultou aos ICTs criarem seus núcleos depois da sua aprovação em 2005. No Nordeste a maioria dos NITs foi criado em 2008.

A ausência de um quadro efetivo no NIT é a principal barreira que os gestores enfrentam para planejar e executar as atividades no núcleo, que tem contado basicamente com a contratação dos bolsistas através dos editais ofertados.

Este estudo possibilitou entender como o NIT atua no processo de gestão do conhecimento em suas atividades visando à transferência de tecnologia para o setor produtivo. Atualmente a área de Engenharia de Produção tem direcionado esforços com estudos ligados a gestão do conhecimento em instituições de ensino e empresas, esta pesquisa poderá direcionar alguns caminhos alternativos de pesquisas, como por exemplo, o NIT como o agente da transferência de tecnologia visando à cooperação entre universidade-indústria.

Neste sentido, vale ressaltar que não existe um modelo único para criação e gestão de conhecimento organizacional para todas as organizações que visam à 
transferência de tecnologia, visto que cada organização tem suas características e uma cultura diferenciada uma da outra. Para encarar estes desafios, os NITs devem ininterruptamente, inovar seus processos e adquirir sempre novos conhecimentos organizacionais visando uma postura empreendedora e inovadora junto ao setor produtivo, à execução, manutenção e, a ampliação dessas ações será fundamental para um maior fortalecimento dos NITs no Estado da Bahia.

\section{REFERÊNCIAS}

AKGÜN, A.E. ; BYRNE, J.; KESKIN, H.; LYNN, G.S.; IMAMOGLU, S.Z. Knowledge networks in new product development projects: a transactive memory perspective. Information and Management, v.42, p.1105-1120, 2005.

ANTONI, M.; NILSSON-WITELL, L.; DAHLGAARD, J. J. Inter-project improvement in product development. International Journal of Quality \& Reliability Management, v.22, no 9, p.876-893, 2005.

BARBOSA, D. B. Direito da Inovação: comentários à Lei 10.973/2004: lei federal da inovação. Rio de Janeiro, RJ: Lumen Juris, 2006.

BARBOSA, F. \& VAIDYA, K. G. Developing technological capabilities: the case of a Brazilian steel company. Technology Management: strategies and applications, v. 3, n. 3, p. 287-298, 1996.

BOURGEON, L. Staffing approach and conditions for collective learning in project teams: The case of new product development projects. International Journal of Project Management, n. 25, p. 413-422, 2007.

BOZARTH, C. ERP implementation efforts at three firms: integrating lessons from the SISP and IT-enabled change literature. International Journal of Operations \& Production Management, v.26, n.11, p. 1223-1239, nov. 2006.

BRASIL. Lei de Inovação Federal no 10.973, de 02 de dezembro de 2004.

Disponível em: < http://www.mct.gov.br/index.php/content/view/8477.html >. Acesso em: 26 fev. 2011.

BRASIL. Lei de Inovação do Estado da Bahia $\mathbf{n}^{\circ}$ 11.174, de 09 de dezembro de 2008. Disponível em: < http://www.uesc.br/nucleos/nit>. Acesso em: 26 fev. 2011.

BRASIL. Lei de Propriedade Intelectual do Brasil ${ }^{\circ}$ 9.279, de 14 de maio de 1996. Disponível em: < http://www.planalto.gov.br/ccivil_03/leis/L9279.htm>. Acesso em: 26 mar. 2011. 
BRITO-CRUZ, C. H. A. Universidade, a empresa e a pesquisa que o país precisa. Revista Humanidades, v.45, p.15-29, 1999.

CARVALHO, F. C. A. Gestão do conhecimento: o caso de uma empresa de alta tecnologia. Dissertação de Conclusão. (Mestrado em Engenharia de Produção) Universidade Federal de Santa Catarina. Florianópolis, 2001.

CASSIOLATO, J. E., \& LASTRES, H. M. M. Tecnoglobalismo e o papel dos esforços de P,D\&I de multinacionais no mundo e no Brasil. Parcerias Estratégicas, parte 4, n. 20, p.1225-1246, 2005.

CERVO, A. L.; BERVIAN, P. A. Metodologia científica. 4. ed.. São Paulo: Makron Books, 1996, $90 \mathrm{p}$.

CHAPMAN, R.; HYLAND, P. Complexity and learning behaviors in product innovation, Technovation, v.24, p.553-561, 2004.

CHESNAIS, F. A mundialização do capital. São Paulo: Xamã, 1998.

CORSO, M.; PAVESI, S. How management can foster continuous product innovation. Integrated Manufacturing Systems, v.11, n. 3, p.199-211, 2000.

CYSNE, F. P. Transferência de tecnologia entre a universidade e a indústria.

Revista eletrônica de Bibl. Ci. Inform. Florianópolis, n. 20, p. 54-74, $2^{\circ}$ semestre 2005.

CUMMINGS, J.L.; TENG, B.S. Transferring R\&D knowledge: the key factor affecting knowledge transfer success. Journal of Engineering and Technology management, v.20, n. 1/2, p.39-68, 2003.

DAVENPORT, T. H.; PRUSAK, L. Conhecimento empresarial: como as organizações gerenciam o seu capital intelectual. 10. ed. Rio de Janeiro: Campus, 2003.

DEITOS, M. L. A Gestão da tecnologia em pequenas e médias empresas.

Cascavel: Edunioeste, 2002.

DEBACKERE, K., \& VEUGELERS, R. The role of academic technology transfer organizations in improving industry science. Research Policy, v. 34, n. 3, p.321-342, 2005.

EDLER, J.; KRAHMER, F. M., \& REGER, G. Changes in the strategic management of technology: results of a global benchmarking study. R\&D Management, v. 32, n. 2, p.149-164, 2002.

EDMONDSON, A.C.; NEMBHARD, I.M. Product development and learning in project teams: the challenges are the benefits. Journal of Product Innovation Management, v.26, p.123-138, 2009. 
ESCORSIM, S. Fatores relevantes no processo de transferência de tecnologia na implementação do sistema de planejamento e controle da produção na indústria Metalgráfica Iguaçu S.A. 2006. 92 f. Dissertação (Mestrado em Engenharia de Produção) - Universidade Tecnológica Federal do Paraná, 2006.

EVANS, P. B.; WURSTER, T. S. Strategy and the new economics of information. Harvard Business Review, v.75, n. 5, p. 71-82, 1997.

FAPESB. Fundação de Amparo à Pesquisa do Estado da Bahia. Disponível em:< www.fapesb.ba.gov.br/.> Acesso em: 26 mar. 2011.

FITZEK, D. Knowledge management in inter-project learning: a systematic attempt of integration. 2. ed. Institut für Technologiemanagement, Universität St. Gallen, 2002.

FLEURY, M. T. L. A relação universidade-empresa: desafios e oportunidades na geração e na disseminação do conhecimento. Revista de Administração da Universidade de São Paulo, v.34, n. 4, p.32-45, 1998.

FLEURY, M. T. L; OLIVEIRA R., Gestão estratégica do conhecimento: integrando aprendizagem, conhecimento e competências. São Paulo: Atlas, 2001.

FRANK, A. G.; ECHEVESTE, M. E. Barreiras de transferência de conhecimentos entre projetos de produtos. Revista Produção Online, v.11, n.1, p.29-53, 2011.

FREEMAN, C. Networks of innovators: a synthesis of research issues. Research Policy, v.20, n. 5, p.499-514, 1991.

GIL, A. C. Como elaborar projetos de pesquisa. 1. Ed. São Paulo: Atlas, 2002. $175 p$.

GOLD, A. H., MALHOTRA, A., SEGARS, A. H. Knowledge management: an organizational capabilities perspective. Journal of Management Information Systems, Armonk, v.18, p.I85-214, 2001.

HSU, I-C. Knowledge sharing practices as a facilitating factor for improving organizational performance through human capital: a preliminary test. Expert System with Application, n. 35, p. 1316-1326, 2008.

INPI. Instituto Nacional de Propriedade Intelectual. Disponível em:< www.inpi.gov.br/.> Acesso em: 26 fev 2011.

KLEINSMANN, M.; BUIJS, J.; VALKENBURG, R. Understanding the complexity of knowledge integration in collaborative new product development teams: a case study. Journal of Engineering and Technology Management, v.27, p. 20-32, 2010. 
LACOMBE, F. Recursos humanos: princípios e tendências. São Paulo: Saraiva, 2005.

LIMA, I. A. Estrutura de referência para a transferência de tecnologia no âmbito da cooperação universidade-empresa: estudo de caso no CEFET-PR. 2004. Tese (Doutorado em Engenharia de Produção) - Universidade Federal de Santa Catarina, Florianópolis, 2004.

MARCONI, M. DE A.; LAKATOS, E. M. Técnicas de pesquisa: planejamento e execução de pesquisas, amostragens e técnicas de pesquisas, elaboração análise e interpretação de dados. 3. ed. São Paulo: Atlas, p.231, 1996.

MARSH, S.J.; STOCK, G.N. Creating dynamic capability: the role or intertemporal integration, knowledge retention and interpretation. Journal of Product Innovation Management, v.23, p.422-436, 2006.

MARTINS, R. de O. Os NITs e as políticas de inovação do MCT. 2010. Disponível em: <http:// www.sct.ce.gov.br/categoria1-fouder/arquivos-anexos/apoio-a-nucleosde-inovacao-tecnologica-nits/6a-reuniao-da-redenit>. Acesso em: 24 jan. 2011.

MATIAS-PEREIRA, J.; KRUGLIASKAS, I. Gestão da inovação: a lei de inovação tecnológica como ferramenta de apoio às políticas industrial e tecnológica do Brasil. RAE - eletrônica, v.4, n. 1, jul./dez. 2005.

MINBAEVA, D. et al. MNC knowledge transfer, subsidiary absorptive capacity, and HRM. Journal of International Business Studies, v.34, p.586-599, 2003.

MOTOHASHI, K. University-industry collaborations in Japan: the role of new technology-based firms in transforming the national innovation system. Research Policy, v.34, n. 5, p.583-594, 2005.

NIEDERGASSEL, B.; LEKER, J. Different dimensions of knowledge in cooperative R\&D projects of university scientists. Technovation, v.31, p. 142-150. 2010.

NONAKA, I; TAKEUCHI, H. The Knowledge-creating company: how japanese companies create the dynamics of innovation. New York: Oxford University Press: 65-80p.

PERIN, M. G. et al. A pesquisa survey em artigos de marketing nos ENANPAD's da década de 90. RIMAR - Revista interdisciplinar de Marketing, v. 1, n. 1, p. 44-59, jan./abr. 2002.

PICININ, C. T.; KOVALESKI, J. L.; REIS, D. R. Aplicação de transferência de tecnologia em práticas de gerenciamento de risco logístico. Revista Produção Online, v.10, n. 1, p.124-141, 2010. 
REIS, Z. C.; MILAN, G. S. Gestão do conhecimento: um desafio a ser administrado. Revista Produção Online, v.9, n.1, p. 73-94, 2009.

ROMANENKO, A.; SANTOS, L. O.; AFONSO, P. A. F. N. A. Application of agent technology concepts to the design of a fault-tolerant control system. Control Engineering Practice, v.15, n. 4, p. 459-469, April 2007.

SANTIAGO JR, J. R. S. Gestão do conhecimento: a chave para o sucesso empresarial. São Paulo: Novatec, 2004.

SEGATTO-MENDES, A. P. A cooperação universidade-empresa como uma das tendências para o crescimento tecnológico das organizações. In: CONGRESSO LATINO-AMERICANO DE ESCOLAS DE ADMINISTRAÇÃO. 37,. 2002, Porto Alegre, RS. Anais... Porto Alegre, RS, 2002.

STEINER, J. E; CASSIM, M. B; ROBAZZI, A. C. Parques tecnológicos: ambientes de inovação. Disponível em:< www.iea.usp.br/artigos.> Acesso em: 14 abr. 2011.

SVEIBY, K. E. A nova riqueza das organizações: gerenciando e avaliando patrimônios de conhecimento. 3. ed. Rio de Janeiro: Campus, 1998.

SUTZ, J. The university-industry-government relations in Latin America. Research Policy, v.29, n. 2, p.279-290, 2000.

SZEZERBICKI, A. S.; PILATTI, L. A.; KOVALESKI, J. L.; FRANCISCO, A. C. Gestão do conhecimento em equipes de alta performance: o caso do Clube Atlético Paranaense. Revista Produção Online, v.6, n. 2, p.01-25, 2006.

TERRA, J. C. C. Gestão do conhecimento: aspectos conceituais e estudo exploratório sobre as práticas de empresas brasileiras. Tese de Conclusão (Doutorado em Engenharia de Produção) - Poli, Universidade de São Paulo, São Paulo, 1999.

TRINDADE, A. L. Metodologia científica: pesquisa e normalização de trabalhos de pós-graduação. Canoas: ULTRACORP, p.50, 2003.

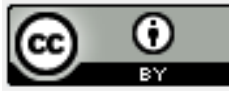

Artigo recebido em 30/05/2012 e aceito para publicação em 01/11/2012. 